\title{
Reflets
}

Revue d'intervention sociale et communautaire

\section{La Semaine nationale du travail social : bilan et perspectives d'un projet d'action communautaire}

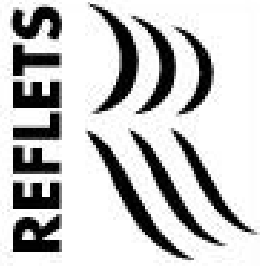

\section{Joscelyne Levesque, Audrey Cayouette Marsh et Jeannine Turpin}

Volume 21, numéro 2, automne 2015

URI : https://id.erudit.org/iderudit/1035439ar

DOI : https://doi.org/10.7202/1035439ar

Aller au sommaire du numéro

Éditeur(s)

Reflets, Revue d'intervention sociale et communautaire

ISSN

1203-4576 (imprimé)

1712-8498 (numérique)

Découvrir la revue

Citer cet article

Levesque, J., Cayouette Marsh, A. \& Turpin, J. (2015). La Semaine nationale du travail social : bilan et perspectives d'un projet d'action communautaire.

Reflets, 21(2), 205-216. https://doi.org/10.7202/1035439ar d'utilisation que vous pouvez consulter en ligne. 


\section{La Semaine nationale du travail social : bilan et perspectives d'un projet d'action communautaire}

Joscelyne Levesque, coordonnatrice des stages et de la formation pratique École de service social, Université d'Ottawa

Avec la collaboration

d'Audrey Cayouette Marsh, étudiante à la maîtrise en service social École de service social, Université d'Ottawa et de Jeannine Turpin, secrétaire à la formation pratique École de service social, Université d'Ottawa

\section{Introduction}

Le présent article porte sur la trajectoire qu'a suivie la Semaine nationale du travail social qui se déroule depuis sept ans maintenant à l'École de service social de l'Université d'Ottawa. Nous en suivons d'abord l'évolution au fil des années en tant que pratique d'action collective tout en identifiant les valeurs qui la transcendent et qui sont étroitement liées à celles mises de l'avant par le travail social. Pour illustrer l'ensemble des démarches et des actions lié à son organisation, nous empruntons certains concepts théoriques se rattachant aux différentes étapes du processus de l'action communautaire. Puis, nous traitons des défis rencontrés et des réponses qui leur ont été apportées. Enfin, nous dressons une appréciation qualitative de l'impact des diverses activités de la Semaine. 
La collaboration d'une étudiante qui a déjà participé aux activités de la Semaine et qui s'est jointe au comité organisateur de la prochaine ajoute une vision expérientielle à la rédaction de cet article.

\section{Les premiers pas... ou la préparation de l'intervention}

L'idée première d'organiser une Semaine nationale du travail social a émergé autour de "discussions de corridor " portant sur le souhait de créer des espaces autres que la salle de classe, espaces pouvant favoriser les échanges entre étudiantes, étudiants, professeures et professeurs dans un climat convivial tout en mettant de l'avant une vision structurelle du travail social, laquelle s'inspire grandement des apports de Jane Addams, entre autres, l'analyse des problèmes sociaux à partir d'oppressions, tels le classisme, le racisme, le capacitisme, l'hétérosexisme, le genrisme ou l'âgisme (Lapierre, et Levesque, 2013; St-Amand, 2011). À titre de coordonnatrice des stages et de la formation pratique, j'entendais les propos des étudiantes et des étudiants en lien avec désir de discuter entre eux de différentes questions sociales, mais aussi avec des professeures ou professeurs, dans un contexte informel favorisant la prise de parole égalitaire. Chaque année depuis 1990, l'Association canadienne du travail social (ACTS) proclame le mois de mars "Mois national du travail social ».Au cours de la première semaine de mars et dans cette même foulée, l'Association des travailleuses et travailleurs sociaux de l'Ontario (ATTSO) organise différentes activités autour d'un thème. À l'École de service social, nous avons choisi d'y tenir au même moment notre Semaine nationale du travail social, mais à partir de thèmes proposés par les étudiantes et les étudiants eux-mêmes.

La mission de l'École de service social repose sur les valeurs de justice sociale et de défense des droits humains, lesquelles rejoignent l'essence même de l'action communautaire, à savoir, la justice sociale, la solidarité et la démocratie (Lavoie, et Panet- 
Raymond, 2014). Ces valeurs ont servi d'assises à la mise sur pied du premier comité organisateur de notre Semaine.Trois personnes motivées par l'expérience étudiante et souhaitant offrir une réponse aux demandes étudiantes composaient ce premier comité, soit la coordonnatrice des stages et de la formation pratique, la personne responsable de l'expérience étudiante à la Faculté des sciences sociales et le responsable des études de premier cycle à l'École de service social. Rapidement, nous sommes passés à l'action et en mars 2009 se tenait à l'École la première Semaine nationale du travail social. Cette activité nous apparaissait comme une possible réponse aux attentes des étudiantes et des étudiants. L'expérience nous a permis de confirmer notre hypothèse et le train a rapidement été remis sur les rails pour une deuxième année, cette fois-ci, de façon plus structurée et mieux organisée : des objectifs clairement définis, une implication étudiante dans l'ensemble du processus organisationnel, des activités souhaitées, des ressources, une appréciation de la Semaine et des suggestions en vue des suivantes.

\section{L'adolescence ou l'exploration de différentes avenues}

Les trois années qui ont suivi ont été des années exploratoires tant sur le plan des thèmes choisis et des personnes impliquées dans l'organisation que sur celui des activités au programme.

Dès les débuts, les thèmes retenus (Pauvreté : il existe des solutions, Dignité et inclusion à travers la défense et le respect des droits la personne et Le pouvoir d'agir des individus et des collectivités au cour du travail social) reflétaient les valeurs propres aux approches structurelles. Ils s'inscrivaient dans une perspective de changement social axé sur une définition des problèmes sociaux selon une analyse critique dénonçant les injustices vécues par plusieurs groupes minoritaires. Rapidement, nous sommes passés d'un discours d'exclusion sociale à celui de dénonciation des oppressions subies par des individus, des groupes ou des collectivités. Nous sommes aussi 
passés de la notion de besoins au concept de droits, des pratiques d'intervention traditionnelles aux pratiques novatrices, dont celles axées sur les arts, ainsi que du savoir professionnel au savoir expérientiel. Le thème annuel se déclinait à travers différentes activités majoritairement réservées aux étudiantes et aux étudiants, mais certaines s'adressant aux partenaires œuvrant dans le champ de l'intervention sociale.

$\mathrm{Au}$ cours des trois années en question, le comité organisateur a exploré différentes avenues et opté pour une planification en différentes formules : panel d'ouverture, conférence thématique, ateliers de formation alternant entre les pratiques usuelles et les pratiques innovantes, dîner-causerie, projection de films à caractère social, théâtre d'intervention, et autres. À la demande des étudiantes et des étudiants, nous avons développé une soirée de réseautage où il leur est possible de rencontrer des organisations œuvrant dans le domaine du travail social. Dans la même foulée, nous avons aussi mis sur pied une soirée de poésie où se côtoient les étudiantes et étudiants, les membres du corps professoral et du personnel de soutien de l'École.

Vu le peu d'intérêt qu'elles ont suscité et qui a entraîné une faible participation, certaines activités ont fait l'objet d'ajustements ou de renouvèlement en profondeur : projection de films suivie de discussions, théâtre d'intervention ou encore atelier de création par l'art autour d'un café.

Cependant, la grande majorité des thèmes et des activités ont été appréciés positivement. Année après année, le panel d'ouverture et les conférences de clôture ont été identifiés comme les coups de cœur tant par les membres du comité organisateur que par les participantes et participants aux différentes activités. Tout en étant arrimée au thème choisi, la formule du panel d'ouverture met de l'avant des gens du milieu de l'intervention. À quelques exceptions près, les conférences de clôture mettent de l'avant le savoir expérientiel, ce qui marque profondément les participantes et les participants. D'ailleurs, nous entendons régulièrement des étudiantes et des étudiants échanger sur les conférences antérieures. L'annexe à la fin de cet article donne un aperçu d'activités inscrites au programme de la Semaine. 


\section{De la maturité au renouvèlement}

Au fil des ans, la planification de la Semaine du travail social s'est modulée non seulement autour de la réalité de la vie étudiante, mais aussi de celle du travail social et de l'environnement sociopolitique dans lequel il évolue. Cependant, nous avons toujours gardé le cap sur les valeurs prônées par les approches structurelles et les pratiques qui en découlent. Les thèmes des trois dernières années en font foi : Travail social et francophonies minoritaires : alliés dans l'action; Travail social : "s'activer " pour la justice sociale; Résister et lutter contre les violences : le rôle du travail social. Nous tenons à maintenir le cap sur les objectifs premiers à savoir favoriser l'expérience étudiante et mettre de l'avant les réalités souvent «à la marge » et les pratiques novatrices qui en découlent.

Avec les années, le comité organisateur a su se renouveler sur le plan du contenu et développer un savoir-faire organisationnel permettant de bonifier le programme des activités. À la fin de chaque Semaine nationale du travail social, ses membres dressent un portrait des défis rencontrés et des éléments de réussite, lequel est pris en compte afin d'apporter des modifications à l'édition subséquente de la Semaine, ou encore d'en conserver les éléments gagnants. Cette rencontre bilan se veut aussi une occasion de célébrer, car « [o]n ne soulignera jamais assez l'importance de ces moments conviviaux qui rapprochent les personnes impliquées dans l'action et resserrent les liens sociaux, contribuant ainsi à donner plus de sens au travail effectué " (Lavoie, et PanetRaymond, 2014, p. 126).

\section{Facteurs de réussite}

Les activités de la Semaine nationale du travail social leur étant d'abord adressées, les étudiantes ou étudiants ont intégré rapidement le comité organisateur. L'École de service social compte deux associations étudiantes, dont les représentantes ou représentants sont les membres piliers du comité organisateur. 
De plus, nous comptons année après année sur l'octroi d'un assistanat étudiant qui joue le rôle de phare dans l'organisation de la Semaine. Cette assistante ou cet assistant a pour mandat de participer activement aux différentes étapes de sa planification et de sa réalisation. Ainsi, la participation active des étudiantes et des étudiants constitue au fil des ans l'un des facteurs de réussite les plus importants de la Semaine, tant dans sa planification ou son déroulement que dans l'évaluation des activités inscrites au programme.

En plus de l'implication étudiante, un autre facteur de réussite concerne le partage des responsabilités et l'espace démocratique au sein du comité organisateur. Le dynamisme, les différentes expertises et la motivation de ses membres à s'impliquer activement ont permis de réaliser l'ensemble des activités et ce, année après année. Les liens et partenariats des membres du comité avec la communauté ont permis d'aller chercher des personnes-ressources tant pour les conférences que pour les ateliers de formation. L'espace démocratique se traduit à travers le processus de prise de décision par consensus tant sur le plan du choix des thèmes et activités que sur celui des échanges entre les membres. Chacun d'eux identifie son degré d'implication selon sa disponibilité et se rend responsable d'une ou de deux activités, dans leur planification comme dans leur déroulement.

Un troisième élément de réussite se rapporte à l'organisation logistique (fréquence et durée des réunions, animation, élaboration d'ordres du jour, rédaction des comptes rendus et réservation des salles) qui favorise une implication soutenue de la part des membres du comité. Souvent, nous accordons peu d'importance à ces éléments, mais nos expériences en organisation d'évènements nous permettent d'assurer que le "contenant » est aussi essentiel que le "contenu ». De plus, le comité organisateur a comme principe de choisir des activités qui amènent les étudiantes et les étudiants à se mettre en action, en marche, pour la justice sociale.

Souvent mise de côté faute de temps, l'appréciation ou l'évaluation est un moment clé dans l'ensemble de l'organisation de la Semaine nationale du travail social. Elle permet de dresser le bilan de l'ensemble du processus de planification et de réalisation 
des activités, le tout étant consigné dans un compte-rendu qui est repris lors de la première rencontre de planification de la Semaine subséquente. Les éléments soulignés dans l'appréciation sont discutés et inclus dans le plan d'action pour l'année en cours. Ce bilan sert aussi "de pont historique " pour les nouveaux membres qui se joignent au comité organisateur dans le cadre de la planification de l'année en cours.

Enfin un dernier élément de réussite que nous souhaitons souligner est l'appui de l'École de service social, celui de la Faculté des sciences sociales et celui du Consortium national de formation en santé, appui financier, mais aussi appui dans la vision de la Semaine nationale du travail social. Sans l'appui de ces organisations, il serait fort difficile de réaliser un projet de cette envergure. Cet appui se concrétise à travers la participation et l'implication des membres du corps professoral de l'École de service social soit à titre de conférenciers ou en inscrivant à l'intérieur des plans de cours enseignés certaines activités auxquelles sont invités à participer les étudiantes et les étudiants.

\section{Nos défis...}

L'un de nos plus grands défis année après année est d'assurer la participation étudiante aux différentes activités. Cette participation varie d'une année à l'autre, d'une activité à une autre. Le contenu des activités, leur calendrier, les conférencières ou conférenciers et les ateliers de formation semblent avoir un impact sur le taux de participation, de même que des facteurs externes à l'organisation de la Semaine, à savoir, les réalités propres aux étudiantes ou étudiants, entre autres, la conciliation travail-étude, les stages ou leur intérêt à participer à des activités autres que leurs cours.

Un autre défi concerne la participation de nos partenaires, tant universitaires que communautaires, aux activités qui leur sont offertes. Peu d'entre eux sont présents. La promotion de la Semaine se fait à travers notre propre réseau de communication. Nous 
devrons explorer d'autres réseaux afin de pouvoir optimiser leur présence. Le comité organisateur souhaite aussi que les activités de la Semaine soient mieux publicisées tant dans les médias universitaires que publics ou privés : journaux, réseaux sociaux, télévision, radio, etc. Depuis plusieurs années, ce point est identifié comme un maillon faible de la planification. Au cours de l'année 2015-2016, des membres du comité organisateur élaboreront un plan de communication. Nous espérons ainsi rejoindre d'autres groupes intéressés à participer à nos activités.

Un dernier défi, et non le moindre, est de s'assurer que la Semaine nationale du travail social maintienne le cap sur son objectif initial : créer un espace d'échanges informels entre étudiantes, étudiants, professeures et professeurs, un espace répondant aux attentes de la communauté étudiante, un espace de pratiques novatrices où la marge devient la norme, où se déclinent les approches structurelles et les valeurs de justice sociale et de changement social à travers les thèmes choisis et les activités, et non recréer un espace cours où se poursuit l'enseignement universitaire.

\section{Un regard étudiant}

"Quelle chance nous avons les étudiantes et étudiants en service social de l'Université d'Ottawa d'avoir une Semaine nationale du travail social où nous sommes invités à participer à une multitude d'activités portant sur des thèmes variés, mais tous actuels.»

«Entre une conférence midi ou une projection de film, la Semaine a souvent été pour moi une opportunité de tisser des liens d'amitié avec les autres étudiants en travail social ainsi que de découvrir notre corps professoral engagé et ouvert. Cette Semaine crée une proximité entre la communauté étudiante et le corps professoral qu'on ne retrouve pas sur les bancs en salle de 
classe en se côtoyant lors des diverses activités. La Semaine offre aussi une soirée réseautage avec un grand nombre d'organismes afin de se faire connaitre par le milieu et surtout de s'informer quant aux emplois disponibles lors de l'obtention de notre diplôme."

«Les thèmes sont porteurs d'espoir, tout en étant revendicateurs. La Semaine nationale du travail social permet de s'outiller davantage afin d'être des candidats intéressants et formés en travail social. Se mobiliser, s'unir, résister, lutter... tous des mots puissants et forts qui nous inspirent dans notre trajectoire d'étude en travail social. Les activités offertes sont variées en terme de temps, de sujets et d'interactions; on peut tous y trouver son compte."

"La Semaine permet aux étudiantes et aux étudiants de découvrir les multiples facettes de ce qu'est le travail social; cette facette nous inspire, nous pousse et nous confronte à une panoplie de rôles qu'on peut être appelé à prendre lors de notre entrée sur le marché du travail. Des témoignages émouvants, des ateliers sur des techniques d'impact, des films-chocs... tout pour nous déstabiliser un peu, et nous confirmer qu'on est bel et bien dans le bon programme d'étude, c'est-à-dire le travail social. »

«C'est toujours plaisant de se retrouver, étudiants, professeurs, membres de la communauté universitaire et partenaires, ainsi que les diverses personnes invitées afin de discuter et d'apprendre sur différents aspects du travail social. La Semaine est à notre couleur comme étudiantes et étudiants et nous rassemble tous autour des mêmes thèmes : justice sociale, respect des droits de la personne et solidarité.» 


\section{Conclusion}

En plus de répondre à son objectif premier de favoriser les échanges entre étudiantes, étudiants, professeures et professeurs dans un cadre informel, la tenue annuelle de la Semaine nationale du travail social permet de mettre de l'avant notre profession selon une perspective qui s'éloigne des modèles d'intervention traditionnels et institutionnels. Elle permet aussi d'offrir un espace d'échanges et de discussions autour de thématiques interpellant différents acteurs : citoyennes et citoyens, intervenantes et intervenants, professeures et professeurs, et autres. La création d'espaces de prise de parole est le premier échelon d'une société démocratique et c'est ce que permet la Semaine nationale du travail social.

\section{Bibliographie}

LAPIERRE, Simon, et Joscelyne LEVESQUE (2013). « 25 ans plus tard... et toujours nécessaires! Les approches structurelles dans le champ de l'intervention sociale ", Reflets : revue d'intervention sociale et communautaire, Vol. 19, $\mathrm{N}^{\circ}$ 1, p. 38-64.

LAVOIE, Jocelyne, et Jean PANET-RAYMOND (2014). La pratique de l'action communautaire, Québec, Presses de l'Université de Québec, 406 p.

SHRAGGE,Éric (2006). Action communautaire : dérives et possibles, Montréal,Éditions Écosociété, 242 p. ST-AMAND, Nérée (2011). Repenser le service social, Ottawa, Merriam Print, 324 p. 


\section{Annexe 1 (a)}

\section{Université d'Ottawa | University of Ottawa}

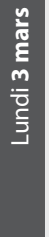

$14 \mathrm{~h} 30$ à $16 \mathrm{~h}$ PANEL D'OUVERTURE FSS4007 (120, Université) " S'activer » pour la justice sociale M. Bill Clennet Mme Ghislaine Sirois Membre du Mouvement étudiant révolutionnaire-Uo Ouvert à la communauté

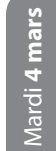

\section{$8 \mathrm{~h} 30$ à 11 h 30}

ATELIER

FSS4004

Travail de rue et réduction des méfaits

par : Marie-Claude Leblanc

Inscription obligatoire et réservée aux étudiantes et aux étudiants en service social

\section{8 h 30 à 11 h 30}

ATELIER

FSS4006

Art et justice sociale

par : Micheline Shoebridge

Dominique Saint-Pierre

Nadia Chaney

Inscription obligatoire et réservée aux étudiantes et aux étudiants en service social

\section{$9 \mathrm{~h}$ à $11 \mathrm{~h}$}

VISITE GUIDÉE

En autobus de certains lieux

importants de la communaute

franco-ontarienne d'Ottawa

Accompagné par le

professeur David Welch

et Philippe Le Voguer

Inscription obligatoire et réservée

aux étudiantes et aux étudiants

\section{SEMAINE NATIONALE DU}

\section{travail social}

Du 3 au 7 mars

Sous le thème:

Travail social : "s'activer "

pour la justice sociale

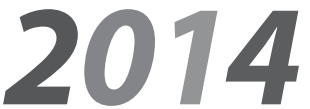

\section{$12 \mathrm{~h}$ à $13 \mathrm{~h}$}

CONFÉRENCE MIDI

FSS4004

Profilage social:

le mal caché de la rue

par : Katharine Larose-Hébert,

Daniel St-Jean,

Émilie Couture-Glassco

Inscription obligatoire

Ouvert à la communauté

\section{$12 \mathrm{~h}$ à $13 \mathrm{~h}$}

CONFÉRENCE MIDI

FSS4006

Nos héritages comparés

du colonialisme

par: Rex Fyles

Inscription obligatoire

Ouvert à la communauté
$13 \mathrm{~h} 30$ à $16 \mathrm{~h} 30$

ATELIER

FSS4004

Média sociaux

par : Isabelle Miron

Inscription obligatoire et réservée

rudiantes et aux étudiants

en service social
13 h 30 à 16 h 30

ATELIER

FSS4006

FEMEN - Le féminisme militant

ou le féminisme dans la rue

par : Neda Topaloski

: Naurence Bergeron$$
\text { Michaud }
$$

Inscription obligatoire et réservé aux étudiantes et aux étudiants en service social
$17 \mathrm{~h} 30$ à 19 h 30

SALON DE RÉSEAUTAGE FSS4004

Rencontre informelle avec des sociales réservée aux étudiantes et étudiants en service social

$19 \mathrm{~h}$ à $21 \mathrm{~h}$ "OSE LA VOIX " Bar L'Avant-Garde, 135 Besserer Soirée de performance artistique francophone : musique, poésie et slam Ouvert à la communaute

\section{$12 \mathrm{~h}$ à $16 \mathrm{~h} \mathbf{3 0}$}

CAFÉ OUVERT

FSS12027

Invitation à créer une œuvre d'art collective, autour d'un breuvage chaud.

\section{$13 \mathrm{~h} 30$ à $16 \mathrm{~h} 30$}

ATELIER

LMX407

Actions politiques

par : Geneviève Latour

Inscription obligatoire et réservée aux étudiantes et aux étudiants en service social
$17 \mathrm{~h} 30$ à $19 \mathrm{~h}$

LANCEMENT

DE LIVRE

FSS4013

Lancement de livre:

"La souffrance à lépreuve

de la pensée "

Co-dirigé par Nicolas Moreau

et Katharine Larose-Hébert

Ouvert à la communauté

\section{$8 \mathrm{~h} 30$ à 11 h 30}

CONFÉRENCE

FSS4007

Enfants transgenres : un organisme communautaire unique en son " genre *

Par: Akiko Asano, Éva Kammer, Annie Pullen Sansfaçon et Mat Asano

Remise de la bourse Michèle-Kérisit

EXPOSITION

FSS4007

Créations artistiques « Violences structurelles *

Ouvert à la communauté

\section{Faculté des sciences sociales}

www.sciencessociales.uOttawa.ca/svs/semaine-svs

\section{Partenaires}

de l'Ontario (ATTSO)

Consortium nationat de formation en santé (CNFS) (volet Université d'Ottawa)

Service de vie communautaire de l'Université d'Ottawa 


\section{Annexe 1 (b)}

\section{Université d'Ottawa | University of Ottawa}

\section{$13 \mathrm{~h}$ à $16 \mathrm{~h}$}

CONFÉRENCE D'OUVERTURE FSS4007 (120, Université)

\section{$13 \mathrm{~h}$ à $14 \mathrm{~h} 30$}

Vivre ensemble... « C'est notre parole qui compte. Nous avons le devoir de parler, de briser le silence! *

Dan Bigras, artiste, cinéaste et porte-parole du Refuge

\section{$14 \mathrm{~h} 30$ à $16 \mathrm{~h}$}

VERNISSAGE

Créer pour lutter : l'Art au service de

la résistance

Ouvert à la communauté

\section{SEMAINE NATIONALE DU}

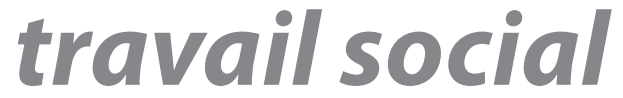

Du 2 au 6 mars

Sous le thème:

Résister et lutter contre les

violences: le rôle du travail social

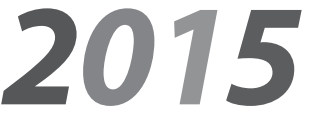

\section{8 h 30 à 11 h 30}

ATELIER

FSS4004

La cyber agression sexuelle faite aux filles et aux femmes

Josée Laramé et Josée Guindon CALACS francophone d'Ottawa

Inscription obligatoire

Réservée à la clientèle étudiante de la Faculté des sciences sociales

\section{$8 \mathrm{~h} 30$ à 11 h 30}

ATELIER

FSS4004

Naviguer nos héritages du colonialisme

Rex Fyles

Inscription obligatoire

Réservée à la clientèle étudiante de la Faculté des sciences sociales

$\mathbf{8 h} \mathbf{3 0}$ à $\mathbf{1 1}$ h $\mathbf{3 0}$

CONFÉRENCE DE CLÔTURE

FSS4007

Les Survivantes

Remise de la bourse Michèle-Kérisit

Un léger goûter sera servi

Ouvert à la communauté

\section{$12 \mathrm{~h}$ à $13 \mathrm{~h}$}

CONFÉRENCE MID

FSS4004

La défense des droits comme dimsion du service socia

Marie Jacque Fortin

Inscription obligatoire

Réservée à la clientèle étudiante

de la Faculté des sciences:

\section{$12 \mathrm{~h}$ à $13 \mathrm{~h}$}

CONFÉRENCE MIDI

FSS4004

La prise de parole publique

pour contrer les vio
faites aux femmes

Isabelle Côté et Simon Lapierre

Inscription obligatoire

Réservée à la clientèle étudiante

de la Faculté des sciences sociales

\section{3 h 30 à 16 h 30 \\ ATELIER \\ FSS4004 \\ Lutter contre la maltraitance \\ envers les ainnées \\ Marie-Eve Manseau Youn \\ csss Cavendish \\ Inscription obligatoire \\ Réservée à la clientèle étudiante de la Facultédes sciences sociales}

\section{$13 \mathrm{~h} 30$ à $16 \mathrm{~h} 30$}

ATELIER

FSS4004

Le travail de rue : la relation d'être

et la qualité du temps perdu

Lovanie Anne Côté, Simon Lemay

et Emilie Couture-Glassco

Inscription obligatoire

Réservée à la clientèle étudiante

de la Faculté des sciences sociales

\section{$16 \mathrm{~h}$ à $19 \mathrm{~h}$}

ATELIER

STEA0150

Rendez-vous avec l'héritage des

pensionnats indiens

Katy Tanguay

Vidéo: : Nous n'étions que des enfants » suivi d'échanges

Inscription obligatoire et réservée
Organisée par :

Les Associations des étudiantes et etudiants en service social, l'École sciences sociales

\section{$17 \mathrm{~h}$ à $19 \mathrm{~h}$}

5 À 7 :

FOIRE AUX RESSOURCES EN SERVICE SOCIAL

FSS4004

Rencontre informelle avec des employeurs et des intervenantes sociales

Un léger goûter sera servi

Ouvert à toute la clientèle étudiante de la FSS

\section{$19 \mathrm{~h}$ à $21 \mathrm{~h}$}

" OSE LA VOIX »

Bar l'Avant-Garde situé au

135 Besserer, Ottawa

Soirée de performance artistique francophone : musique, poésie et slam

Un léger goûter sera servi

Ouvert à la communaute

\section{Partenaires}

Association des travailleuses et travailleurs sociaux de l'Ontario (ATTSO)

Consortium national de formation en santé (CNFS) (volet Université d'Ottawa Service de vie communautaire de l'Université d'Ottawa

\section{Faculté des sciences sociales} sciencessociales.uOttawa.ca/svs/semaine-svs 